\title{
Fracture and strain rate behavior of airplane fuselage materials under blast loading
}

\author{
J. Mediavilla Varas ${ }^{1, a}$, F. Soetens ${ }^{2}$, E. Kroon ${ }^{1}$, J.E. van Aanhold ${ }^{2}$, O.R. van der Meulen ${ }^{1}$, and M. \\ Sagimon $^{1}$ \\ 1 TNO-Defense Security and Safety, Rijswijk, The Netherlands \\ 2 TNO Built Environment and Geosciences, Delft, The Netherlands
}

\begin{abstract}
The dynamic behavior of three commonly used airplane fuselage materials is investigated, namely of A12024-T3, Glare-3 and CFRP. Dynamic tensile tests using a servo-hydraulic and a light weight shock testing machine (LSM) have been performed. The results showed no strain rate effect on A12024-T3 and an increase in the failure strain and failure strength of Glare-3, but no stiffening. The LSM results on CFRP were inconclusive. Two types of fracture tests were carried out to determine the dynamic crack propagation behavior of these materials, using prestressed plates and pressurized barrels, both with the help of explosives. The prestressed plates proved to be not suitable, whereas the barrel tests were quite reliable, allowing to measure the crack speeds. The tougher, more ductile materials, Al2024-T3 and Glare-3, showed lower crack speeds than CFRP, which failed in a brittle manner.
\end{abstract}

\section{Introduction}

Triggered by the growing terrorist threat, the EU VULCAN project [1] aims at strengthening airborne structures under blast and fire. Within this framework, TNO's mission is to investigate the behavior of airplane fuselage materials under blast loading. An explosion is a highly dynamic event and the fuselage material behaves in a different manner as compared to a static load; stress waves, inertia, temperature and strain rate effects take place. An experimental-numerical research has been setup, focusing on two aspects: dynamic strength and fracture behavior. Three typical airplane fuselage materials have been studied: Aluminum 2024T3, Glare-3, CFRP.

The aim is of this paper is to characterize the dynamic behavior of these type of materials, in order to better understand their behavior and hence optimize the design of structures against blast of other types of dynamic loading events.

\section{Dynamic tensile tests}

High strain rate tests have been performed using two different two different test machines:

1. A servo-hydraulic high-speed single shot test machine.

2. A so-called lightweight shock testing machine (LSM).

The servo-hydraulic test machine was used in $[2,3]$ to measure the dynamic properties of S2 glass fibers, Glare-3 and A12024-T3. Stresses are directly computed from the measured force, and the strain and strain rates are obtained from digital processing of high speed camera images. The LSM, on the other hand, is normally used for verifying equipment's resistance to underwater shock induced deck

\footnotetext{
a e-mail: jesus.mediavillavaras@tno.nl
} 
motions on board of naval ships. Stresses are computed from the mass and the acceleration of the clump mass and strains are measured by means of strain gauges. The servo-hydraulic setup is preferred to the LSM, since it allows to reach higher strain rates, up to $1000 \mathrm{1} / \mathrm{s}$, and the tests are very reproducible, with less dynamic oscillations. On the other hand, the LSM allows for testing substantially larger specimens, for example structural details, at dynamic loading rates [4]. Fig. 1 shows the two setups.
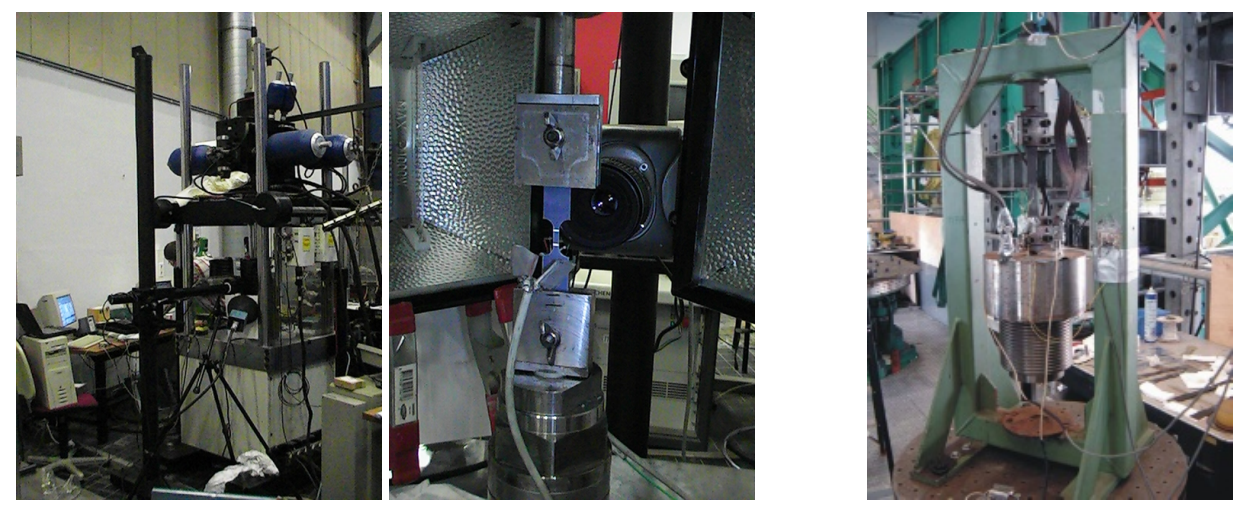

Fig. 1. (left) servo-hydraulic high-speed machine setup; (middle) sample and high speed camera; (right) LSM setup.

\subsection{Aluminum 2024T3}

The specimens tested with the servo-hydraulic machine are $3 \mathrm{~mm}$ wide and $1 \mathrm{~mm}$ thick. Strain rates near 1000 1/s are attained. No strain rate dependency was observed. See Fig. 2. The average failure strain rate was $\epsilon_{f}=0.2$ and the failure strength $\sigma_{f}=550 \mathrm{MPa}$, irrespectively of the strain rate.

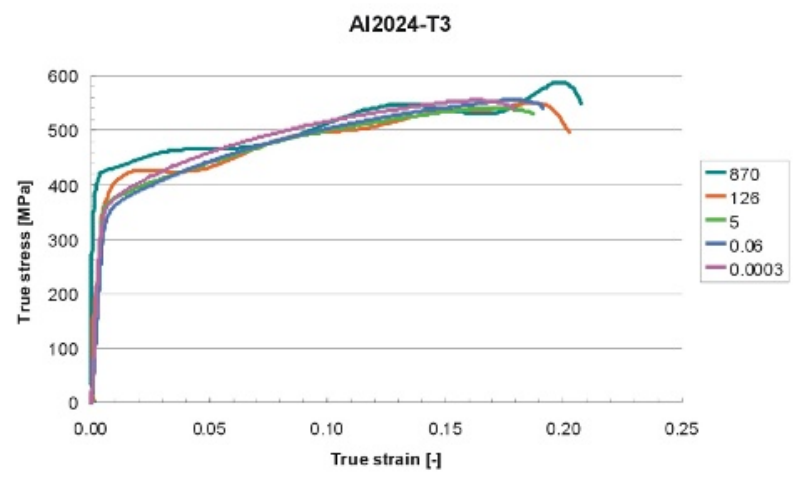

Fig. 2. Stress-strain curves at different strain rates, with servo-hydraulic high-speed machine.

The specimens tested with the LSM were $40 \mathrm{~mm}$ wide and $1.0 \mathrm{~mm}$ thick. The average strain rate was $30 \mathrm{l} / \mathrm{s}$. The interpretation of the results is not straightforward, since the measurement reflected dynamic structural effects (not shown). The dynamic LSM tests showed an average failure strain $\epsilon_{f}=$ 0.18 and the average failure strength $\sigma_{f}=407 \mathrm{MPa}$. Fig. 3 shows that failure occurs along the maximum shear direction, at $45^{\circ}$ from the loading direction, in contrasts to the smaller specimens tested 
with the servo-hydraulic machine, which failed in the direction perpendicular to the loading direction. Note that the specimen geometries and hence the stress states and the failure modes are different. Hollow specimens, with a hole diameter of $5 \mathrm{~mm}$, were also tested to show the effect of high stress triaxialities on reducing the failure strain. This was indeed confirmed by experiments, which showed a lower failure strength and strain, $\sigma_{f}=279 \mathrm{MPa}$ and $\epsilon_{f}=0.017$ respectively. The hollow specimens failed in the normal to the loading direction.

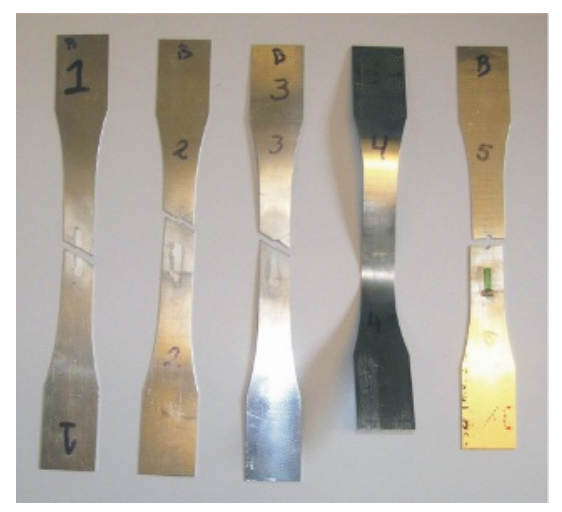

Fig. 3. Aluminum specimens after failure, using LSM machine.

\subsection{Glare}

Static and dynamic tests on Glare specimens were performed using the the servo-hydraulic high-speed and the LSM machine. Two types of Glare were tested with the servo-hydraulic machine: Glare 3-2/1$0.3,1.1 \mathrm{~mm}$ thick; and Glare 3S-3/2-0.3, $2.0 \mathrm{~mm}$ thick. The stacking sequence was $\mathrm{Al} / 0 / 90 / \mathrm{Al}$ and $\mathrm{Al} / 0 / 90 / 90 / 0 / \mathrm{Al} / 0 / 90 / 90 / 0 / \mathrm{Al}$ respectively. The specimens were $3 \mathrm{~mm}$ wide. Fig. 5 shows the stressstrain curve for the Glare 3S-3/2-0.3 specimens. The maximum strain rate reached for Glare 3-2/1-0.3 and Glare 3S-3/2-0.3 equals approximately 500 1/s and $3001 / \mathrm{s}$ respectively. Due to the contribution of the $\mathrm{S} 2$-glass fibers, which are highly strain rate sensitive, significant improvement in straining capacity is seen for Glare 3 with increasing strain rates. Consequently, a significant strength increase is obtained for higher strain rates. No strain rate effect is observed in the yield stress and stiffness. Table 1 reports the failure strain and failure strength at different strain rates. The LSW Glare specimens were Glare $3-3 / 2-0.5,2.0 \mathrm{~mm}$ thick, with a $\mathrm{Al} / 0 / 90 / \mathrm{Al} / 90 / 0 / \mathrm{Al}$ stacking. For the same reasons as argued for the Aluminum tests, servo-hydraulic tests are preferred (stress-strain curves are not shown). Fig. 5 serves to illustrate how failure occurs, normal to the loading direction, and with delamination of the different layers.

\begin{tabular}{|l|c|c|c|c|c|c|c|c|c|c|}
\hline$\dot{\epsilon}$ & 0.001 & 0.01 & 0.1 & 1 & 10 & 100 & 200 & 300 & 500 & 1000 \\
\hline$\epsilon_{f}(\%)$ & 4.4 & 4.4 & 4.6 & 5.2 & 5.7 & 6.3 & 6.5 & 6.6 & 6.7 & 6.9 \\
\hline$\sigma_{f}(\mathrm{MPa})$ & 814 & 814 & 880 & 966 & 1054 & 1142 & 1169 & 1185 & 1204 & 1231 \\
\hline
\end{tabular}

Table 1. Failure strain and failure strength versus strain rate. Glare 3S-3/2-0.3. 


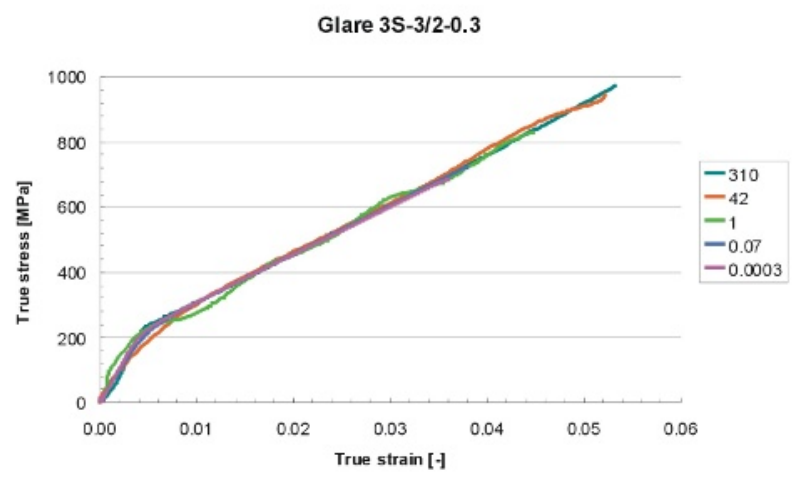

Fig. 4. Stress-strain curves obtained with servo-hydraulic high-speed machine.
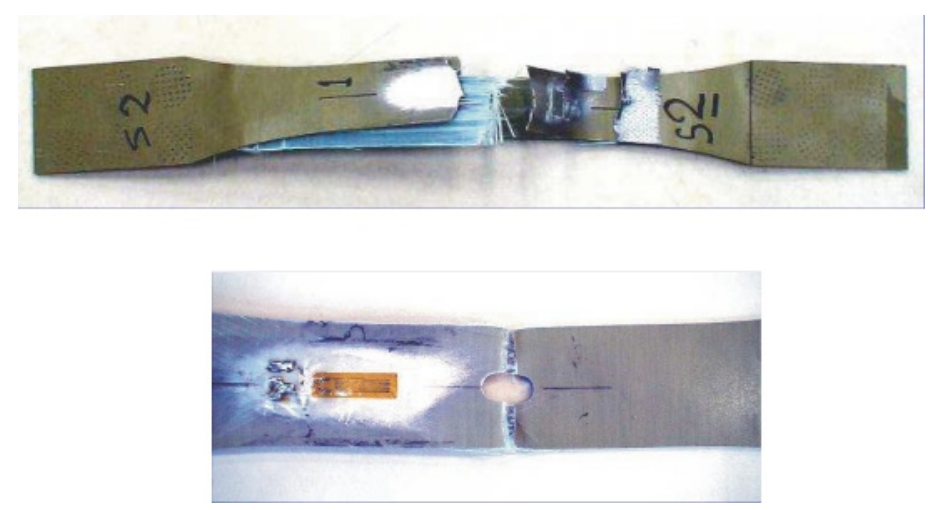

Fig. 5. Glare specimens after failure, using LSM machine.

\subsection{CFRP}

Tensile tests on CFRP were performed using the LSM machine. The specimens are $40 \mathrm{~mm}$ wide and 2 mm thick, made of 10 layers [+/-45,0/90,90/0,+/-45,0/90]. The material used is T300 3k PW (E-765). The average strain rate was only 10 1/s. Fig. 6 shows that in contrasts with Glare and aluminum, which are ductile materials and fail at the middle of the specimen, failure of CFRP may happen at different locations, due to the wave propagation. The stress-strain results of these tests are difficult to interpret (not shown) and is recommended to perform servo-hydraulic high-speed tests in the future.

\section{Dynamic fracture experiments}

Two types of fracture experiments have been performed:

1. Prestressed plate tests.

Flat plates of dimensions 800-1600 (w-1) were prestressed at different stress levels, 100 and 200 $\mathrm{N} / \mathrm{mm} 2$. Crack propagation was triggered by creating a notch $(200 \mathrm{~mm}$ long) in the middle of the plate, by means of an explosive charge. Crack propagation was recorded using high speed camera recordings, which allowed to compute the crack speed. It turned out that this setup was not suitable for studying crack propagation for a number of reasons: unknown extent of the damage near the explosive charge, out of plane movement of the plates due to the explosive load; asymmetric crack propagation. Fig. 7 shows the hydraulic used in the prestressed plate tests, and some snapshots during mode-I crack propagation of the Aluminum, Glare and CFRP specimens. 


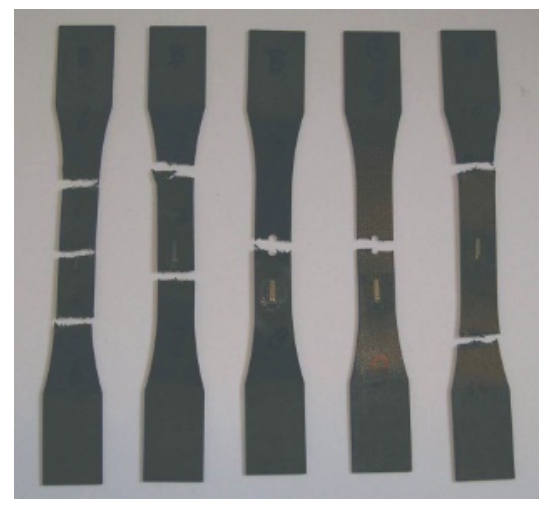

Fig. 6. Specimens after failure, using LSM machine.

2. Pressurized barrel tests.

In the barrel tests, a barrel with a prenotch (56 mm long) is pressurized, and crack propagation is triggered by the explosion of a TNT charge placed inside, in the middle of the barrel. The barrel dimensions are: $1.2 \mathrm{~m}$ by $1 \mathrm{~m}$ (diameter $\mathrm{x}$ height). The top and bottom of the barrel are made of massive steel plates, which are firmly fixed relative to one another in the vertical direction. The skin of the barrel is fixed to the end plates with bolts. The explosive charge is spherical and has a mass of $54 \mathrm{gr}$. To simulate flying conditions, the barrel was pressurized at $200 \mathrm{kPa}$. The prenotch is taped off to avoid depressurization. Upon pressurization, the explosive is detonated, triggering crack propagation.
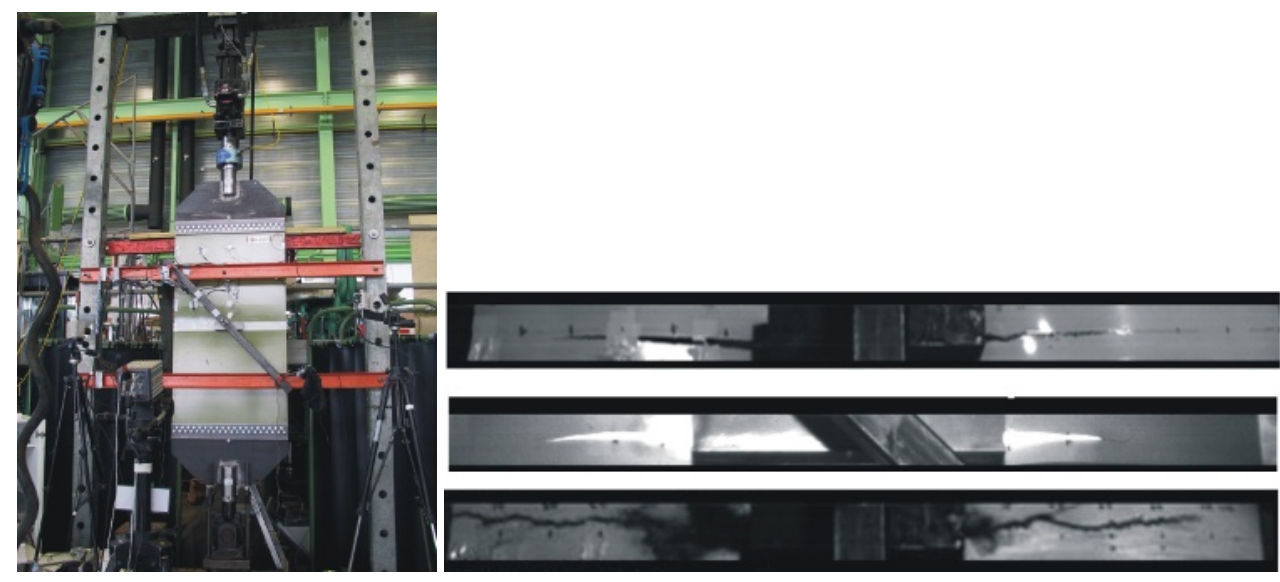

Fig. 7. (left) prestressed plate setup; (right) high speed camera snapshots during crack propagation for Aluminum (above), Glare (middle) and CFRP (below).

Fig. 9, Fig. 10 and Fig. 11 show the barrel after the explosion, a close-up of the crack and the crack speed versus crack length for the Aluminum, Glare and CFRP barrels respectively. Aluminum and Glare failed in a ductile manner, with cracks perpendicular to the maximum hoop stress (mode-I), and relatively low crack speeds. The average crack speeds were $300 \mathrm{~m} / \mathrm{s}$ and $200 \mathrm{~m} / \mathrm{s}$ for aluminum and Glare respectively. This agrees with the higher toughness of Glare. CFRP shows brittle facture, manifested by a very high crack speed, $2500 \mathrm{~m} / \mathrm{s}$, crack bifurcation and zig-zagging.

The lowest and highest crack speeds were found for Glare and the CFRP respectively, since these are the toughest and the most brittle materials. These experiments have been used to validate and im- 

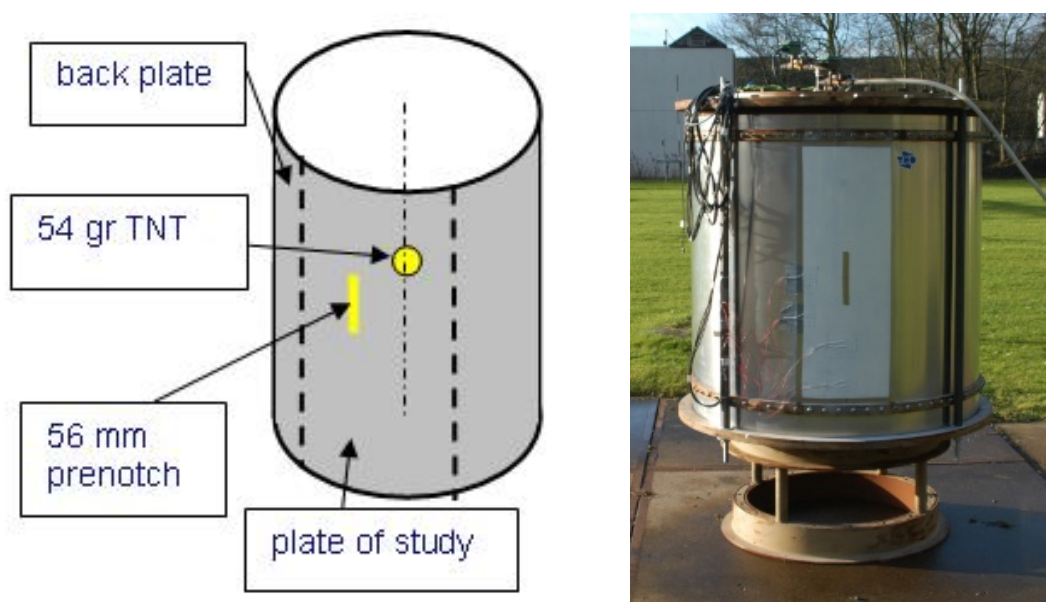

Fig. 8. (left) sketch of barrel test set-up; (right) photograph of barrel test set-up.

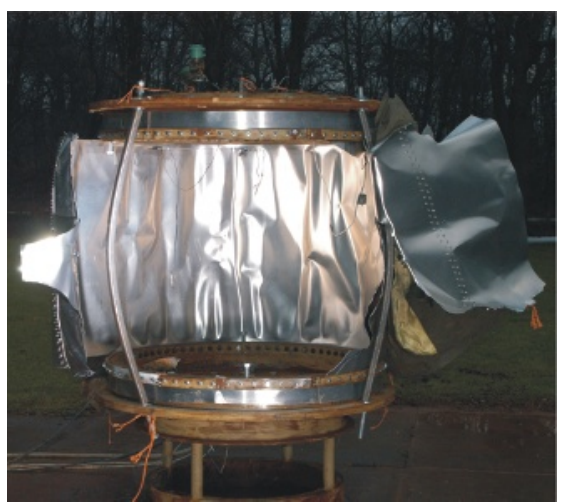

(a)

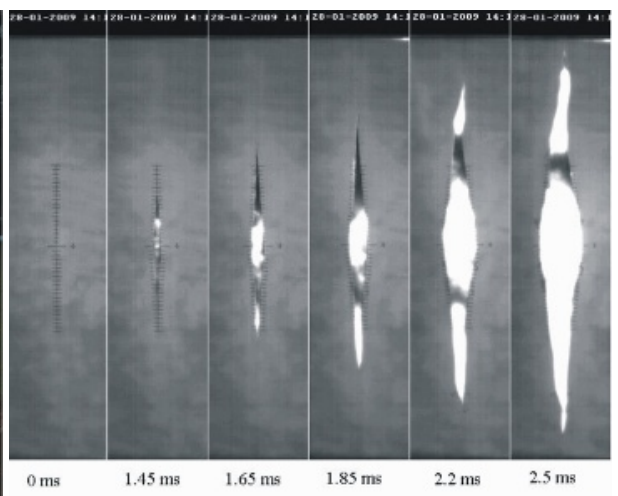

(b)

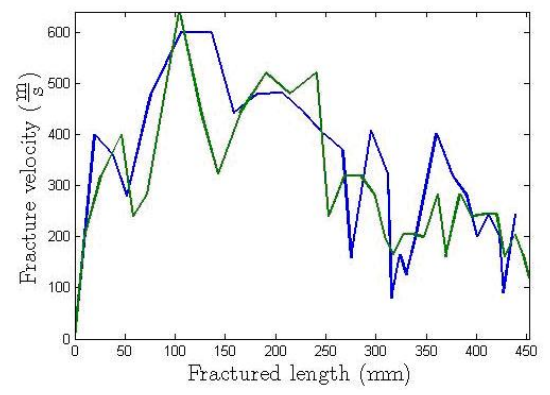

(c)

Fig. 9. (a) aluminum barrel after explosion; (b) high speed camera snapshots during crack propagation; (c) crack speed versus crack length. 


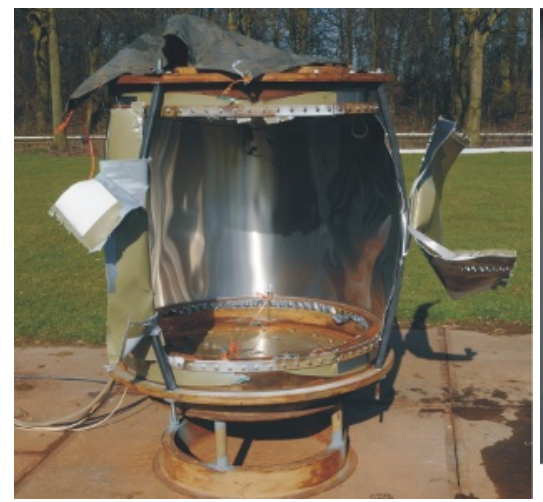

(a)

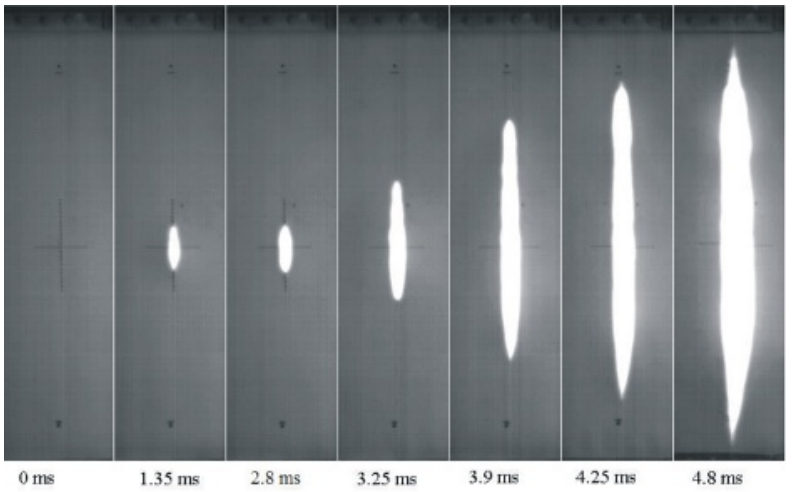

(b)

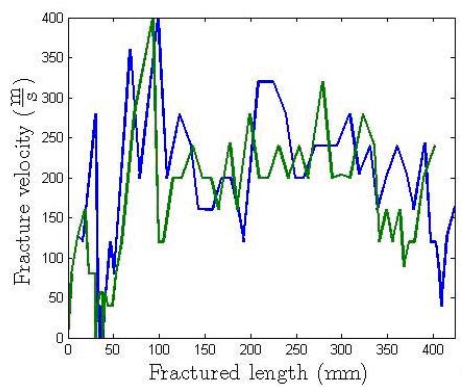

(c)

Fig. 10. (a) Glare barrel after explosion; (b) high speed camera snapshots during crack propagation; (c) crack speed versus crack length.

prove existing finite element fracture models (cohesive models) [5]. By an inverse modeling technique, it was shown that the fracture toughness increases under dynamic conditions.

\section{Conclusions}

To characterize the mechanical behavior of airplane fuselage materials (Al2024-T3, Glare-3, CFRP) under dynamic loading, dynamic tensile tests and fracture tests have been performed. The main conclusions are summarized below.

- Dynamic tensile tests.

1. AL2024T3 shows no strain rate effect, constant failure strain and failure strength upon different strain rates. Glare-3 shows an increase in failure strain and failure strength at high strain rates, while the stiffness and yield stress remain constant. The CFRP tests with the LSM machine were inconclusive and further experiments must be done.

2. The servo-hydraulic tests are preferred over the LSM tests, since the corresponding equipment produces excellent and highly repeatable results without superimposition of stress waves. Furthermore, higher strain rates can be attained in comparison with the LSM testing method.

- Dynamic crack propagation tests.

1. The prestressed plate tests prove not to be suitable for monitoring crack propagation, due to a lack of symmetry, undesirable effects caused by the explosive charge (out of plane displacement and unknown extent of damage around the crack tips).

2. The barrel tests on the other hand allow to monitor the mode-I crack propagation and measure the crack speed of the different material tested, and it is very useful in order to validate numerical dynamic fracture models. 


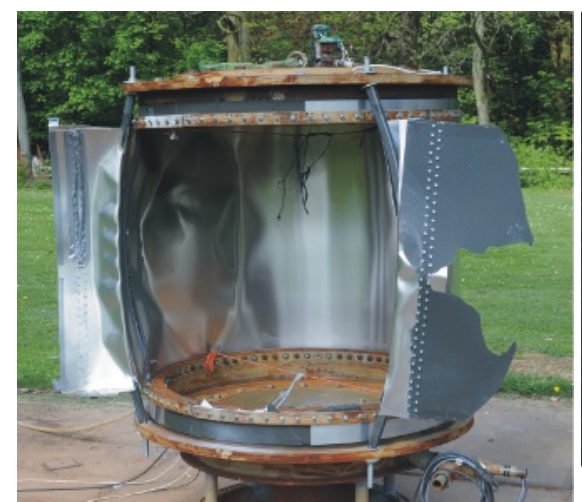

(a)

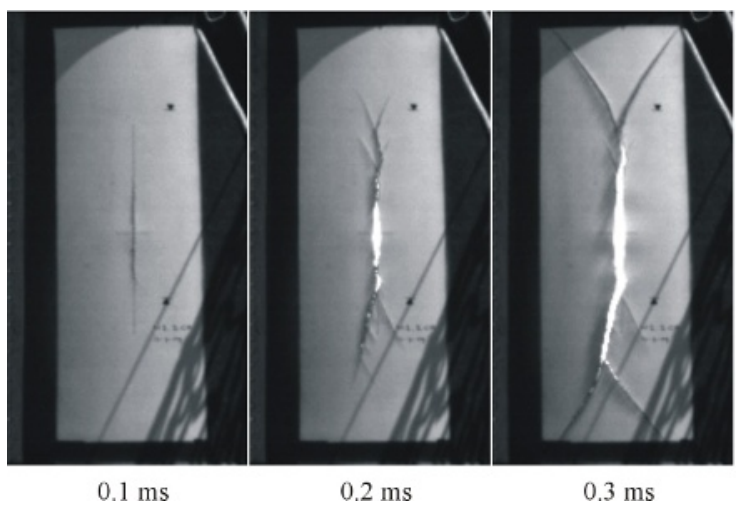

(b)

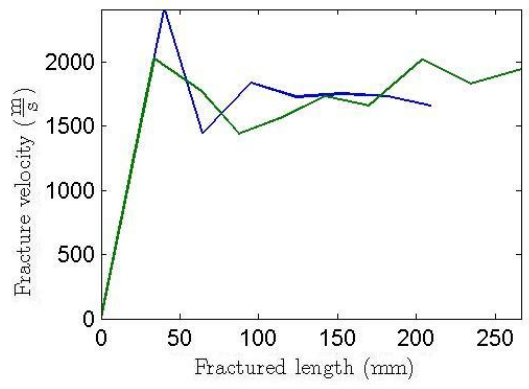

(c)

Fig. 11. (a) CFRP barrel after explosion; (b) high speed camera snapshots during crack propagation; (c) crack speed versus crack length.

3. Glare and Aluminum displayed a ductile behavior during crack propagation, while CFRP confirmed its brittle behavior.

\section{Acknowledgments}

The authors would like to acknowledge the partners of the EU project VULCAN and TNO-DSS for their contribution to this research.

\section{References}

1. European-Comission, Vulcan-strep-fp6 (2005).

2. E. Kroon, Dynamic tensile strength of fm94 s2-glassfiber composite, Tech. Rep. TNO-DV 2009 IN630, TNO (december 2009).

3. G. Pape, Low and high strain rate tensile test results of al2024-t3, fm94 s2-glassfiber composite and glare, Tech. Rep. 2009003, DTRL (December 2009).

4. J. Aanhold, A. Weersink, J. Ludolphy, Dynamic testing of adhesive joints using a shock testing machine, in: 69th Shock \& Vibration Symposium, St. Paul MN, USA, 1998.

5. J. Mediavilla Varas, R. van der Meulen, J. Weerheijm, Dynamic crack propagation of prestressed metallic structures: an experimental-numerical approach, in: 17th European Congress of Fracture, Brno, Czech Republic, 2008. 